\title{
Une thèse CIFRE en collectivité territoriale : concilier la recherche et l'action?
}

\section{Camille Rouchi}

\section{(2) OpenEdition}

\section{Journals}

Édition électronique

URL : https://journals.openedition.org/cdg/1523

DOI : $10.4000 /$ cdg. 1523

ISSN : 2107-7266

Éditeur

UMR 245 - CESSMA

\section{Référence électronique}

Camille Rouchi, « Une thèse CIFRE en collectivité territoriale : concilier la recherche et l'action? », Carnets de géographes [En ligne], 11 | 2018, mis en ligne le 15 septembre 2018, consulté le 20 mai 2021. URL : http://journals.openedition.org/cdg/1523; DOI : https://doi.org/10.4000/cdg.1523

Ce document a été généré automatiquement le 20 mai 2021.

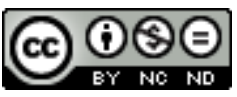

La revue Carnets de géographes est mise à disposition selon les termes de la Licence Creative Commons Attribution - Pas d'Utilisation Commerciale - Pas de Modification 4.0 International. 


\title{
Une thèse CIFRE en collectivité territoriale : concilier la recherche et l'action?
}

\author{
Camille Rouchi
}

1 Depuis la fin des années 2000, plusieurs travaux ont été menés sur la recherche en contrat CIFRE (Conventions Industrielles de Formation par la REcherche ${ }^{1}$ ), dans les diverses disciplines de sciences humaines et sociales à travers le prisme de la recherche partenariale (Audoux et Gillet, 2011; Gillet, 2008; Collectif, 1991; Seferdjeli et Stroumza, 2011), salariée en générale (Perrin-Joly, 2010) et surtout de la rechercheaction (Amado et Levy, 2005 ; Liu, 2005) qui se définit comme :

2 "Une approche de recherche, à caractère social, associée à une stratégie d'intervention et qui évolue dans un contexte dynamique (...) fondée sur la conviction que la recherche et l'action peuvent être réunies. Selon sa préoccupation, la recherche-action peut avoir comme but le changement, la compréhension des pratiques, l'évaluation, la résolution de problèmes, la production de connaissances ou l'amélioration d'une situation donnée » (Barbier, 1996).

3 Ces travaux, menés principalement en sociologie (Galio, 2008) ou dans les études de communication (Morillon et Gardère, 2009 ; Foli et Dulaurans, 2013), n'échappent pas à la discipline géographique, notamment lorsqu'elle rend compte des territoires politiques et des stratégies des organisations publiques.

4 C'est dans ce sens, que nous souhaitons apporter notre analyse quant à la particularité de ce contrat tripartite engageant le doctorant à la fois auprès de son laboratoire de recherche, de l'Agence Nationale de la Recherche et de la Technologie (ANRT) et d'une collectivité territoriale. Car, si les contrats CIFRE en sciences humaines auprès d'un opérateur public se multiplient, il n'est pas toujours évident pour le doctorant de se situer non pas dans un double, mais dans un triple contexte académique, professionnel et éminemment politique du monde territorial : un triple contexte qui entraine autant de responsabilités que de contradictions. Il s'agira en outre, de mener d'une part, une approche réflexive sur les enjeux constitutifs d'une thèse CIFRE, et d'aborder 
l'élaboration du terrain et l'intérêt des différents partis. Et d'autre part, de communiquer sur un « retour d'expérience » spécifique à sa réalisation dans la fonction publique territoriale.

5 S'engager dans une thèse CIFRE confronte rapidement le doctorant à un certain nombre de questionnements: Comment élaborer au quotidien la possibilité de la recherche-action? Comment adopter un regard réflexif sur un terrain contraint à l'observation participante (Bogdan, Taylor, 1975)? Comment ne pas basculer vers le rapport d'expertise et la prospective, dans la production d'une thèse scientifique ? Dans ces conditions, nous nous poserons comme question principale: Peut-on concilier la recherche en sciences humaines et l'action en collectivité territoriale dans le cadre d'une thèse CIFRE ?

6 Pour répondre à cette question, nous avons choisi de questionner les principaux conflits rencontrés dans les différents milieux (professionnels, politiques et académiques) fréquentés au cours des trois étapes majeures de la production scientifique : la définition et le traitement de l'objet de recherche, l'accès aux données et la restitution des travaux. Les conflits nous permettant de révéler, ce que peuventêtre une thèse en sciences humaines, ses finalités et sa distinction du rapport ou de l'expertise. La notion de conciliation nous permettant d'interroger la capacité de ces deux univers à se rapprocher pour un le temps et pour des intérêts a priori convergents.

\section{Traitement de l'objet de recherche et problématique de l'engagement}

\section{Des avantages d'une thèse CIFRE...}

7 Nombreux sont les avantages d'une thèse CIFRE pour un doctorant notamment en sciences humaines, où il est parfois difficile d'obtenir des financements. En outre, accédant au statut de salarié, le doctorant une fois contractualisé se retrouve protégé et se voit attribuer : salaires et prestations sociales conséquentes, et notamment le droit au chômage pour un avenir post-thèse parfois compliqué. Côté recherche, cette possibilité d'immersion professionnelle est éminemment pratique pour l'obtention des informations et des données relatives à la thèse. Côté carrière, l'opportunité d'être engagé au sein d'une structure publique ou privée renforce les compétences du doctorant et son appréciation de l'environnement professionnel, il permet également d'affirmer ou d'infirmer un choix d'orientation, voire d'obtenir un contrat supplémentaire.

8 Du côté de la structure employeuse ensuite, l'engagement en collectivité ou en entreprise auprès du doctorant permet à la fois l'obtention d'un travail de recherche approfondi et concerté en amont ; et, il est une manière efficace pour l'établissement de porter une collaboration de recherche avec un laboratoire public. Ajoutons par ailleurs que le dispositif CIFRE permet à l'entreprise de bénéficier d'une aide financière non négligeable, puisque l'établissement reçoit pendant trois ans de l'ANRT, au nom de l'Etat, une subvention annuelle de 14000 euros.

9 Mais l'emploi d'un doctorant peut répondre à d'autres avantages. Dans mon cas puisqu'il s'agit d'un retour d'expérience -, mon intégration au sein d'un Conseil 
départemental des Yvelines en juillet 2014, m'a permis d'accéder au plus près de mon terrain : le territoire yvelinois et son rapport tantôt d'inclusion et d'exclusion à la Métropole du Grand Paris alors en construction. Cela m'a permis, en outre, de débattre régulièrement au sein de mon service d'accueil, consacré à la culture (spectacle vivant, lecture publique) sur des questions à la fois réflexives et prospectives, relatives à la question du rayonnement culturel métropolitain, et du non-rayonnement d'une offre jugée périphérique, tenant en grande partie de la ruralité. Etre partie-prenante des réflexions est d'autant plus intéressant si, comme cela a été mon cas, le doctorant est invité à enrichir le débat de par ses recherches et parfois de porter des propositions d'action, dans un contexte où le service d'accueil n'a ni le temps, ni les moyens humains de s'y consacrer. De plus, mon orientation géographique est dès le départ un atout pour un environnement professionnel porté sur une expertise disciplinaire (théâtre, musique, arts-plastiques, lecture...), aujourd'hui confrontée à une réforme territoriale majeure (Acte III de la décentralisation) qui impose d'orienter les politiques publiques sur une action de plus en plus territorialisée.

\section{... Aux conflits dans l'engagement : la temporalité, l'utilité, l'intégrité}

De ce contexte avantageux intervient alors de nombreux conflits et contradictions, tant $\mathrm{du}$ fait de l'intégration dans l'environnement professionnel, que de l'intégrité professionnelle, c'est-à-dire de la conformité du travail fourni aux exigences de la recherche scientifique du doctorant.

11 De mon expérience, le premier conflit est apparu d'ordre politique. Perçue comme une employée à part entière, ma venue a été l'occasion privilégiée de valoriser les positions politiques d'une direction, d'une collectivité, ou plutôt de son élu. En effet, la signature de mon contrat a été autant une opportunité, qu'un prétexte pour le Conseil départemental de créer une "cellule économie" au sein de la Direction de la culture, légitimant la nouvelle politique culturelle portée par l'élu en place, privilégiant l'évaluation des politiques menées (et en particulier les ponctions budgétaires). Cette cellule qui a ainsi pendant un temps eu sa place dans l'organigramme de la collectivité, permettait de justifier en interne l'emploi d'un doctorant d'une part, et de répondre à un positionnement politique. C'est là qu'est apparue la première difficulté dans l'élaboration de l'objet de recherche en thèse CIFRE : une confusion dans l'engagement. En effet demeurera tout le long de la recherche, une confusion quant aux missions à réaliser dans l'entreprise ou la collectivité. Et ce, d'autant plus lorsque l'on réalise une thèse de sciences humaines dont le sujet de recherche n'est pas l'unique motivation du contrat, du moins du côté professionnel.

12 Ainsi la question est de savoir : comment servir d'une part l'intérêt scientifique et l'objet de recherche pour lequel on s'est investi, et d'autre part, les intérêts d'une collectivité avec qui on est engagé et sous contrat? Car la collectivité veut avoir un rôle actif dans l'appréciation des travaux de recherche: contrôler le sujet et sa méthodologie, surtout dans une institution où l'on est tenu à la discrétion de sujets politiques et réflexions sensibles. D'autant, qu'elle souhaite aussi avoir la possibilité de renégocier régulièrement, au fil des actualités et des questionnements institutionnels, des commandes parfois très éloignées du projet de départ.

Il est apparu au fil de mon immersion, un second conflit tout à fait lié au premier : un conflit se matérialisant par une contradiction forte entre deux temporalités : celle de la 
recherche et celle de l'employeur, et plus spécifiquement celle d'une collectivité territoriale. Tandis que l'objet et le traitement de la recherche impose nécessairement un temps long puisque l'élaboration d'une thèse se fait en trois ans au minimum (sans compter les temps annexes mais tout autant nécessaires: la réponse aux appels à communication, la présentation à des colloques, la rédaction d'articles, voire l'enseignement), les temps électoraux eux, mais aussi des évaluations (généralement annuelles) sont bien plus courts. En effet, d'une part, la collectivité veut pouvoir justifier politiquement de l'utilisation des deniers publics octroyés au doctorant contractualisé, dans un contexte de plus en plus contraint des finances publiques. D'autre part, et parallèlement, la collectivité souhaite obtenir des résultats réguliers, dans la mesure où elle souhaite procéder à une évaluation annuelle de ses agents, dont le doctorant-CIFRE ne se distingue pas juridiquement. Ainsi cette contradiction peut engager des renégociations en continu au profit du temps court - celui de l'administration publique - qui doit pouvoir commander, obtenir et évaluer sur des périodes plus restreintes.

Dans le cadre de ma thèse, ces exigences s'ajoutent encore à deux conflits " d'intérêt " entre les deux mondes - académique et professionnel. D'abord, le sujet de recherche et son traitement sont évolutifs au cours des trois ans, disons par nécessité intellectuelle : le processus de recherche nous amène à nous orienter vers de nouvelles questions et à adapter l'approche méthodologique. Et, de leur côté, les missions et la position au sein de la hiérarchie de la collectivité d'accueil, peuvent également évoluer au gré des restructurations internes à l'organisation, et des stratégies politiques. Pour reprendre mon expérience, la fameuse "cellule économie » mise en place au sein de la Direction de la Culture du Département, qui devait au départ investir la question de l'économie culturelle autant que celle de la territorialisation, et de ce fait servir en partie l'élaboration du sujet de thèse, a été remise en cause. Cet espace reconnu par l'organigramme, qui me permettait autant de justifier ma présence, que mon action au sein de l'institution, était un espace source de réciprocité et de réflexivité nécessaire à mes questionnements. N'existant plus, l'équilibre des intérêts réciproques est rompu, et la collectivité qui signe l'avènement de sa nouvelle politique et bouleverse l'organisation et les fonctions de ses agents devient une menace pour un contrat à durée déterminé qui apparait relativement commode à briser. Il est bon de rappeler que cette thèse signée "avant la tempête ", à parcouru les péripéties des réformes institutionnelles (loi Maptam, loi NOTRe) et les baisses de dotations qui sont à l'œuvre en particulier depuis 2015.

Dans l'urgence et la menace, les conséquences peuvent alors toucher à la fois l'intégrité personnelle et professionnelle $\mathrm{du}$ doctorant (l'objectivité scientifique) en déconsidérant progressivement ou radicalement la question de recherche pour s'investir dans un nouveau rôle dans l'organisation. De cette manière, le statut particulier du doctorant CIFRE, ne l'empêche pas de se soumettre comme tout employé de la collectivité aux exigences des élus et à la courte durée des politiques. Ainsi, les financements du terrain de recherche, dans le domaine public (et il en va de même dans le domaine privé) posent la question des contraintes liées à l'élaboration et au traitement de l'objet de recherche, et interrogent la posture du chercheur dans l'institution.

Être engagé par son terrain d'observation pose donc une série de questions d'ordre pratique (la justification du statut), méthodologique (la justification politique du projet 
de recherche, mais aussi nous le verrons l'accès aux données) mais surtout épistémologique (les objectifs de le recherche en sciences humaines pour l'employeur). L'une des principales difficultés est alors d'articuler une double position : «être engagé dans son terrain, mais aussi par son terrain » (Alam, Gurruchaga et O'Miel, 2012), dans la mesure où les observations sont menées à la fois dans l'institution et sur l'institution (au regard de leurs actions sur le territoire d'analyse).

\section{La question de l'accès aux données}

\section{Du partage des données...}

Travailler au sein d'une collectivité apparait d'abord comme une chance pour pouvoir commencer l'observation et le recueil d'informations, et ce d'autant plus dans le cadre d'une recherche en géographie où la question du terrain apparait autant essentielle que l'accès aux données pour la constitution d'un corpus cartographique. Par ailleurs, trois années passées dans une collectivité semblent constituer un moment privilégié d'immersion sur le terrain où l'on peut se rapprocher de la méthode ethnographique, en négociant par exemple l'intérêt de nous laisser accéder à d'autres niveaux pertinents d'informations et d'analyse, à d'autres services, à d'autres directions dans l'organisation. Et pourtant, l'accès au «terrain ", même lorsqu'on y est engagé, peut s'avérer problématique et parfois de manière multiple.

Ce qu'il faut d'abord constater - et la problématique est similaire dans le domaine de la recherche -, c'est qu'il n'est pas très répandu dans l'institution publique, de partager ses données. Et ce, malgré la mode actuelle pour l'émergence des open data (libre accès aux données), open access (libre accès aux productions scientifiques) ou open process (libre usage des publications et des données), qui correspondent à ce mouvement planétaire issu des Etats-Unis, en raison duquel gouvernements, administrations et collectivités, tendent à rendre publiques certaines de leurs données. Cela fonctionne en France avec le site data.gouv.fr, et on voit que la logique open data oblige à modifier le comportement des administrations qui l'ont adopté. Le mouvement des humanités digitales s'efforce depuis plus de dix ans de multiplier les initiatives en ce sens (Bouchet, Carnino et Jarrige, 2016). En témoignent notamment des portails open data de plusieurs collectivités territoriales (Métropole, Conseil régional, etc.) d'ores et déjà en fonction, opérationnels et portés par des entrepreneurs champions de l'économie numérique comme Gilles Babinet, actuellement le digital champion ${ }^{2}$ de la France auprès de la Commission européenne, responsable des enjeux de l'économie numérique et chargée de promouvoir les avantages d'une société numérique en France. Un mouvement qui favorise par ailleurs, l'émergence de start-up technologiques qui collectent, analysent et enrichissent ces données, et pousse à ce que certains appellent «l'hypnose numérique» (Reuss, 2013) dans l'enseignement supérieur et la recherche, en témoigne notamment l'ouverture de MOOC ("Massive Open Online Course »), dont l'essor récent en 2002 participe d'une transition sans précédent concernant le bien commun: dans un mouvement ascendant, des externalités locales aux externalités globales. 


\section{...aux conflits d'intérêts chez les administrés comme chez les scientifiques}

19 Si ces nouvelles tendances sont fortement débattues dans la sphère publique, elles n'influencent actuellement que peu les collectivités territoriales et, le Conseil départemental au sein duquel je suis employée ne fait pas exception. Ainsi aujourd'hui ni la recherche, ni l'ensemble des collectivités ne sont passées à l'open data, même si cela fait partie des grands discours, voire de grands projets. Et la raison est assez simple, elle est une question de conflit d'intérêts qui n'épargne ni les scientifiques dans le domaine de la recherche, ni les collectivités dans le domaine des institutions, c'est-àdire qu'il apparait chez de nombreux laboratoires et agents, qui tous ont à accomplir une fonction d'intérêt général, des intérêts personnels ou de service (publications, programmes de recherche, promotions, etc.) parfois en concurrence avec leurs missions attribuées. Les conflits d'intérêt sont également internes aux institutions, qui caractérisent les rapports hiérarchiques: entre directeur, sous-directeur ou chef de services. Des rapports d'intérêts qui sont finalement à la fois représentatifs du manque flagrant de transversalité entre les directions et les services, et représentatifs de ce qu'on pourrait appeler la guerre des chefs", soit la concurrence parfois plus personnelle que professionnelle entre les services d'une même institution. Cela est encore une chose que l'on peut retenir de mon retour d'expérience : il m'est plus que difficile d'aborder d'autres services que celui qui m'accueille. La crainte étant pour ma Direction (ou plutôt pour ma supérieure hiérarchique), que je court-circuite les stratégies et les actions menées par la collectivité et notamment les actions menées dans les services dont elle n'a pas autorité. De la même manière, lorsque j'ai souhaité obtenir des informations, ou des détails budgétaires auprès d'autres collectivités de ma région-terrain, la suspicion de mes interlocuteurs m'a obligé à ne pas mentionner mon poste au sein du Conseil départemental et la particularité du contrat CIFRE. La mise en concurrence fut donc palpable même entre institutions, et ce sans doute, d'autant plus fortement dans un contexte important de restructuration politique.

Pour éviter ce genre de situation, il s'agirait à la fois de moderniser les rapports hiérarchiques au sein des collectivités et de favoriser le travail en transversalité. Cela permettrait en outre, et je m'appuie encore sur mon terrain, de constater que des diagnostics territoriaux sont réalisés sous plusieurs formes, par les différentes institutions territoriales (intercommunales, départementales, régionales et autres) et au sein des institutions par différentes directions et services. Cela permettrait aussi d'obtenir des données chiffrées qui ne soient pas seulement agglomérées à l'échelle régionale ou traitées sous divers angles selon les besoins des directions, ce qui oblige le chercheur à un long travail de récupération de données des différents services, échelons institutionnels et organismes extérieurs (sociétés civiles, organismes d'Etat, cabinets privés, etc.). Cela irait par ailleurs dans le sens d'une transparence démocratique, sur l'utilisation des fonds publics et les actions menées.

21 Ainsi, cette seconde approche du terrain qui est celle de l'accès aux données, interroge finalement autant le milieu académique et notamment la condition du jeune chercheur à l'ère numérique, que le milieu professionnel et spécifiquement de la fonction publique territoriale aujourd'hui plus que jamais décentralisée (notamment grâce à la poursuite de l'intercommunalisation, loi NOTRe). Mais, cette question interroge aussi la 
question du citoyen dans l'obtention de données publiques, à l'heure où l'on prône la transparence politique.

\section{Restitutions et valorisation des travaux}

\section{De la mise en tensions d'objets différents...}

Nous avons vu dans la première partie de cet article que, si le doctorant CIFRE doit répondre à la demande de l'entreprise ou de la collectivité contractante, il doit également gérer le fait qu'au cours de ces trois années contractualisées, une évolution de la demande sociale est possible, au bon vouloir de ses supérieurs et des élus, tous soumis au turn-over électoral traditionnel et à l'actualité institutionnelle. Il faut ajouter à cela que les demandes formulées par la collectivité portent parfois sur des objets sans rapport direct entre eux. Il apparait alors difficile de relier les apports des différents travaux dans une réflexion cohérente, tout en raccrochant les résultats de ces travaux aux questions de la recherche académique. Une des manières de concilier productions professionnelles et productions scientifiques serait alors d'utiliser une partie des activités menées au sein de la collectivité dans la réalisation de certaines étapes de la thèse.

Quoi qu'il en soit, le terrain met en tension des objets différents, comme la commande en milieu professionnel, généralement ponctuelle et précise, et la situation de travail qui s'oppose souvent à l'objet d'analyse sur lequel il s'agit de s'investir et d'adopter un regard distancié. Tout au long de mon contrat CIFRE, il m'a été demandé par exemple de réaliser des études, plus ou moins éloignées de mon objet de recherche : étude sur l'emploi culturel dans les Yvelines, étude sur l'entrepreneuriat culturel à l'échelle de la métropole parisienne, étude sur les potentialités touristiques de l'offre culturelle yvelinoise. Je tiens à ajouter, que ces commandes ont été saisies, et en partie du moins, négociées avec mon employeur qui se languissait sans doute de ne pas avoir rapidement de résultats « exploitables » pour la refonte de ses objectifs politiques.

Ainsi, il nous apparait que le terrain met en tensions des logiques d'action différentes: il semble tout à fait contradictoire de résoudre un problème selon le vœu de l'employeur, tout en approfondissant un sujet selon le vœu du chercheur. Et dans ce sens, la question des thèses CIFRE en sciences humaines est absolument problématique, et elle l'est sans doute moins pour les disciplines scientifiques. Cela étant, la restitution des résultats d'une recherche dans le milieu professionnel dépasse la simple transmission d'informations. Intermédiaires ou finaux, ces résultats apportent des informations et des connaissances inédites à la collectivité et de nouvelles perspectives sur les thèmes analysés. Il permet de questionner pour l'apprenti-chercheur autant l'objet de la commande que le contenu.

Même si, bien généralement, le travail de thèse apparait comme une option plutôt que comme finalité pour l'employeur, la production scientifique doit à ce titre servir l'institution. La thèse, loin du rapport professionnel, doit pourtant dans l'esprit des commanditaires institutionnels, conforter l'organisation dans ce qu'elle croit et fait: apporter une plus-value qui ne soit pas une remise en question. C'est là qu'apparait toute la difficulté du doctorant-CIFRE : apprendre à concilier les désirs et les réalités et à affirmer surtout, sa position de chercheur, sa distanciation et son objectivité. Le terrain doit être à la fois une source d'action dans le domaine professionnel (expertise, 
prospective) et dans le domaine académique (point étape, méthode de recherche qualitative et/ou quantitative), mais aussi l'objet d'une production réflexive (observation, restitution) à la fois nécessaire pour les deux parties tout au long du contrat, et généralement source de tensions jusqu'à la restitution.

Malgré l'ensemble des difficultés constatées, il semble possible de concilier un travail de thèse en s'adaptant aux deux logiques du monde professionnel et du monde académique, mais le traitement de l'objet de recherche, comme la restitution, doivent passer par des étapes et des actions d'ordres différents qu'il s'agit de confronter si possible en amont.

Puisqu'il apparait difficile en début de carrière, d'être à la fois un cadre opérationnel, un consultant et un apprenti chercheur dans une organisation, il faut prendre en compte une équation complexe et la présence d'injonctions paradoxales dans ces différents rôles professionnels et sociaux. Il s'agit donc d'imaginer un compromis professionnel particulier, imposé par notre terrain qui nous permet à la fois de mener des activités pour l'entreprise ou la collectivité d'accueil et d'obtenir une certaine liberté pour effectuer nos travaux de recherche.

\section{...A la distance du chercheur}

La clef de ce débat est alors sans aucun doute la réflexivité, qui apparait comme l'élément fédérateur entre le doctorant et la collectivité. Puisqu'il faut bien entendu affronter les interrogations épistémologiques et le terrain sur le mode réflexif (Bertrand, 2014; Dumez, 2012), et ce, d'autant plus dans le cadre des recherches en sciences humaines, où l'objectivité et la distanciation (Paillé, 2010 [2006]) sont davantage sujets à critique, la collectivité ou l'entreprise employeuse a tout intérêt à s'emparer de ses formes de restitutions. La réflexivité n'apparait pas seulement comme une démarche méthodologique, elle est une opportunité en tant que démarche politique. En effet, dans la mesure où la réflexivité questionne la valeur sociale de ses choix d'objet et ses conséquences, elle est éminemment politique, et à ce titre elle apparait autant nécessaire pour le doctorant que pour l'institution qui l'emploie.

Ainsi la question de la restitution des travaux de recherche répond d'abord à l'enjeu premier d'un contrat CIFRE, c'est à dire la production d'une thèse, mais en dépasse largement cette ambition. Car communiquer sur ses recherches, c'est adopter un regard réflexif sur l'organisation contractante : cela apparait comme la condition qui permet l'entente et la compréhension de différents milieux scientifique et professionnel, les attentes et les besoins de chacun des acteurs sur un objet.

Nous conviendrons qu'aujourd'hui de plus en plus, le milieu de la recherche se rapproche de l'entreprise, des organisations ou de la société civile. Les disciplines de sciences humaines sont à ce titre particulièrement concernées, puisqu'elles ajoutent par leur pluridisciplinarité, des enjeux croisés (économiques, géographiques, sociologiques, politiques...), qui complexifient d'autant plus les relations entre les acteurs au sein des institutions et les partenariats avec le domaine privé. Ainsi, au regard des projets de recherche-action, la restitution et la valorisation d'un objet de recherche permet de lier besoins et compétences à la fois scientifiques et professionnels.

31 Nous pouvons ajouter, puisque la réflexivité est un enjeu politique, que les finalités de la recherche présentent des enjeux forts de responsabilité sociales: les résultats 
peuvent avoir des portées multiples tant en termes d'avancées scientifiques que d'intérêt social. Et surtout, dans le domaine public, une portée politique dans la mesure où ses restitutions peuvent aider à la prise de décision, voire engager des fonds publics.

\section{Conclusion}

32 A ce stade, ce retour d'expérience nous permet de constater qu'être engagé par son terrain, c'est se confronter à la contradiction des temporalités et des intérêts: les temps longs de la recherche et les temps plus courts du domaine professionnel; le besoin de données et la difficulté d'en obtenir l'accès ; la production scientifique et la restitution d'un rapport adapté aux besoins et à la lecture professionnelle. Le terrain met en tensions des objets différents, comme la demande professionnelle, la situation de travail ou la distance critique qui s'oppose souvent à l'objet d'analyse sur lequel il s'agit de s'investir et d'aborder d'un regard distancié. En effet, les objectifs de ces deux mondes s'avèrent au départ presque opposés: l'un cherche un surplus d'innovation pratique et un partenariat prestigieux, et l'autre, lui préfère l'ancrage académique et le travail scientifique original, permettant une avancée des connaissances dans sa discipline. Par ailleurs, soulignons qu'à la difficulté du doctorant CIFRE à jongler entre les mondes académique, professionnel et politique, s'ajoute leur faible reconnaissance post-thèse par le milieu académique, dans un contexte de forte valorisation des étudiants normaliens, agrégés et allocataires-moniteurs ; cette situation incarnant sans doute une sourde méfiance entre les deux parties.

Ainsi, les principaux conflits exposés ne limitent pas seulement la conciliation entre deux univers, car se rapprocher pour un temps peut leur permettre de se retrouver autour d'intérêts a priori convergents, mais la recherche et l'action n'ont pas in fine vocation à être conciliables. Dans cette perspective, le travail de thèse en sciences humaines se distingue irrémédiablement du rapport ou de l'expertise. La conciliation est alors possible que si les temporalités et les objectifs divergents sont entendus dès le départ par les différents signataires du contrat, et s'il est clarifié l'objet même d'une thèse de science humaine. Il apparait aussi urgent de clarifier auprès des partenaires financiers du doctorant, que celui-ci n'est ni un stagiaire, ni un consultant, mais bien un étudiant dont la position tantôt d'observation, tantôt participante se justifie avant tout pour son apport scientifique, ce qui ne contredit aucunement son utilité sociale.

Pour autant, et malgré l'ensemble des difficultés rencontrées, le contrat CIFRE, nous apparait comme un moyen essentiel d'entente et de compréhension entre le doctorant et le monde des acteurs, de la même manière qu'il est un outil de valorisation et de promotion de la recherche dans le monde de l'entreprise. Il est ainsi une opportunitépasserelle entre deux univers sans doute trop séparés. Et, ce rapprochement est d'autant plus important que la responsabilité sociale du chercheur en entreprise est de faire reconnaitre par l'organisation contractante l'utilité sociale et de la pratique en sciences humaines. Car après tout « la recherche-action ambitionne de forger un code commun aux praticiens et aux chercheurs, ayant fait le constat que ce code commun n'existait pas. ", écrivait Michel Bataille (1984, p150). La recherche-action, la porosité des recherches et des réflexions avec le monde professionnel et la société civile, tous ces éléments appréhendés en termes d'enjeux et de responsabilité sociale, nous apparaissent encore comme des compétences nécessaires pour consolider un partenariat de recherche, faire connaitre la production et l'intérêt de la recherche 
française, et parallèlement pour construire une frontière professionnelle entre métier de chercheur et métier de consultant.

\section{BIBLIOGRAPHIE}

Amado G.,Levy A., 2005, La recherche-action Perspectives internationales, Eska, Paris.

Audoux C., Gillet A., 2011, « Recherche partenariale et co-construction de savoirs entre chercheurs et acteurs : l'épreuve de la traduction », Revue Interventions économiques, mai.

http://interventionseconomiques.revues.org/1347

Barbier R., 1996, « La recherche-action », Anthropos, Paris.

Bataille M., Une recherche-action coéducative, Université de Toulouse-Le-Mirail, 1984, p. 150.

Bertrand, E.,2014, "Le dialogisme expérimentiel : d'une conformation à une coformation critique", Education permanente, mars, $\mathrm{n}^{\circ} 198$.

Bogdan R., Taylor S.,1975,Introduction to qualitative research methods, in Lapassade G., L'ethnosociologie, Paris, Méridiens Klincksieck, 1991.

Bouchet T., Carnino G.,JarrigeF., 2016, « L'Université face au déferlement numérique ", Variations, $\mathrm{n}^{\circ} 19$.

http://variations.revues.org/740

Collectif, 1991, Entreprises et chercheurs, à la recherche d'un partenariat, Paris, L'Harmattan (série Cahiers de Cargèse).

Dumez, H., 2012, « Les trois risques épistémologiques de la recherche qualitative », Le libelio d'AEGIS, vol. 8, n 4-Hiver, pp. 29-33.

Foli O., DulauransM., 2013, « Tenir le cap épistémologique en thèse Cifre. Ajustements nécessaires et connaissances produites en contexte ", Études de communication, $n^{\circ} 40$, pp 59-76.

Gaglio G., 2008, En quoi une thèse CIFRE en sociologie forme au métier de sociologue ? Une hypothèse pour ouvrir le débat, Socio-logos, $\mathrm{n}^{\circ} 3$.

http://socio-logos.revues.org/2093

Gillet, A., 2008, « La recherche en entreprise dans le cadre d'un doctorat : un mariage entre recherche académique et utilité sociale ", in Pichault F. et al.ii (dir.), La recherche-intervention peut-elle être socialement responsable ?, Paris, éd. Vuibert, pp. 27-45.

Liu M., 2005, Fondement de la recherche-action, L'Harmattan, Paris, 351p.

Morillon L., Gardere E., 2009, » Le doctorant CIFRE en communication des organisations : un jeune chercheur entre implication et distanciation ", in Loneux C., Parent B. (dir.), Communication des organisations, recherches récentes, Tome 2, L'Harmattan, Paris, pp. 185-196. Paille, P., 2010, La méthodologie qualitative : Postures de recherche et travail de terrain, Paris, Armand Colin, 238 p. 
Perrin-Joly C., 2010, » De la recherche salariée en France : lien de subordination et liberté de la recherche », in SociologieS, La recherche en actes, Expériences de recherche, décembre 2010.

http://sociologies.revues.org/3380

Reuss R., 2013, Sortir de l'hypnose numérique, Vergne-Cain B.,Rudent G. (Trad.), éd. des îlots de résistance, $150 \mathrm{p}$.

Seferdjeli L., Stroumza K., 2011, » Partenariat de recherche en Hautes écoles spécialisées Santé Travail social de Suisse occidentale », in SociologieS, Dossiers, Les partenariats de recherche, octobre.

http://sociologies.revues.org/3622, dernière consultation le 13 septembre 2012.

\section{NOTES}

1. Depuis plus de 30 ans, le dispositif CIFRE - Conventions Industrielles de Formation par la REcherche - subventionne toute entreprise de droit français qui embauche un doctorant pour le placer au cœur d'une collaboration de recherche avec un laboratoire public. Les travaux aboutiront à la soutenance d'une thèse en trois ans. Les CIFRE sont intégralement financées par le ministère de l'Enseignement supérieur et de la Recherche qui en a confié la mise en œuvre à l'ANRT.

2. cf. La Liste des Digital Champions en 2015 par pays, sur le site de la Commission européenne, http://ec.europa.eu

\section{RÉSUMÉS}

Depuis la fin des années 2000, les conventions industrielles de formation par la recherche (CIFRE) se multiplient auprès des doctorants en sciences humaines. Pourtant, il n'est pas toujours évident pour le doctorant de se situer dans un double contexte académique et professionnel qui entraine autant de responsabilités que de conflits. Peut-on concilier la recherche en géographie, et plus globalement en sciences humaines et l'action en collectivité territoriale dans le cadre d'une thèse CIFRE? A travers un "retour d'expérience " spécifique à la réalisation d'une thèse dans la fonction publique territoriale, nous questionnerons les principaux conflits rencontrés dans les deux milieux professionnels et académiques au cours des trois étapes majeures de la production scientifique : la définition et le traitement de l'objet de recherche, l'accès aux données et la restitution des travaux. Les conflits nous permettant de révélerce que peuvent-être une thèse en sciences humaines, ses finalités et sa distinction du rapport ou de l'expertise, et ainsi d'envisager la possibilité d'une conciliation entre recherche et action.

In France, since the late 2000's, Industrial agreements of training by research (CIFRE) have increased for thesis students in Humanities and Social Sciences. However, it is a sensitive task for the student to find a balanced position between the academic and professional work, because having responsibilities and high expectations on both ends can lead to conflicts of interest. So how can research and professional activity conciliate in the framework of a CIFRE PhD? Through a specific "feedback" on the achievement of a $\mathrm{PhD}$ in the french public sector, we will question 
the main conflicts encountered in both the academic and the professional fields. We will see three major steps of the scientific production: the definition and treatment of the research object, the access to data, and the work restitution. Conflicts allow us to reveal what a Humanities and Social Sciences thesis can be, where lie its purposes are and what its distinctions from report or expertise are.

Keywords : Action research, thesis, feedback, Humanities, Social Sciences, methodology

\section{INDEX}

Thèmes : Carnets de terrain

Keywords : Keywords: Action research, thesis, feedback, Humanities, Social Sciences, methodology

Mots-clés : recherche-action, CIFRE, retour d'expérience, sciences humaines, terrain, méthodologie

\section{AUTEUR}

\section{CAMILLE ROUCHI}

Docteur; laboratoire EIREST, Université Paris 1 Panthéon-Sorbonne

camille.rouchi@hotmail.com 